\title{
Dilemmasituation: Nächtlicher Unruhezustand beim älteren Patienten
}

Differenzialdiagnose und Therapie

Dirk Schwerthöffer, Bastian Fatke, Hans Förstl

\begin{abstract}
Ältere Menschen, vor allem mit demenziellen Erkrankungen, sind nachts häufig unruhig, können sich dadurch selbst gefährden oder ihr Umfeld beeinträchtigen. Psychopharmaka sind oft nicht oder paradox wirksam und haben erhebliche Neben- und Wechselwirkungen. Was ist hier zu tun? - Der Beitrag bildet den Auftakt einer Reihe von Situationen, für die es keine oder gegensätzliche Therapieempfehlungen gibt und so die Behandler vor ein Dilemma stellen.
\end{abstract}

\section{Differenzialdiagnose}

\section{Es muss nicht immer Demenz sein}

Bei nächtlichen Unruhezuständen muss zunächst eine Reihe von häufigen Ursachen und Differenzialdiagnosen erwogen werden, die jeweils unterschiedliche diagnostische und therapeutische Maßnahmen erfordern $(\triangleright$ Tab. 1).

\section{Merke}

Erst nach Ausschluss der Differenzialdiagnosen kann man zu der plausiblen Annahme gelangen, dass ein akuter oder chronisch rezidivierender, agitierter Verwirrtheitszustand bei zugrunde liegender Demenz vorliegt.

\section{Verwirrtheitszustände erkennen}

Bei Verdacht auf einen Verwirrtheitszustand kann zur Objektivierung ein kurzer Test angewendet werden, der die Domänen Wachheit, Orientierung, Aufmerksamkeit und Symptomfluktuation überprüft ( $\triangleright$ Tab. 2).

\section{Therapie}

\section{Nichtpharmakologische Maßnahmen haben Vorrang}

Medikamente sind wichtig bei der Behandlung, können aber selbst sogar Ursache nächtlicher Verwirrtheitszustände sein [2].

\section{Merke}

Bevor pharmakologische Maßnahmen zum Einsatz kommen, müssen zuerst alle nichtpharmakologischen Möglichkeiten ausgeschöpft werden.
- Abb. 1 zeigt, dass die nichtpharmakologischen Milieuund Pflegeinterventionen absoluten Vorrang besitzen und notwendig sind, um diagnostische und therapeutische Schritte einzuleiten.

\section{Vor- und Nachteile pharmakologischer Therapien abwägen}

Kognitive Defizite bis zur Demenz sind die häufigsten Ursachen für nächtliche Unruhezustände bei älteren Patienten [3]. Für einige Verhaltenssymptome, die im Rahmen von demenziellen Erkrankungen auftreten, existieren leitlinienbasierte pharmakologische Empfehlungen. So kann zur Behandlung von Apathie mittelfristig die Verordnung von Antidementiva erwogen werden. Bei Agitiertheit können sedierende Antidepressiva [3] und bei Aggressivität möglichst nebenwirkungsarme Neuroleptika verordnet werden [4]. Für die Therapie nächtlicher Unruhezustände liegen für diese Patientengruppe jedoch keine evidenzbasierten Empfehlungen vor. Stattdessen erfolgt hierfür in der Praxis überwiegend eine symptomorientierte Off-Label-Behandlung mit Hypnotika (meistens Benzodiazepine) oder Antipsychotika.

\footnotetext{
Merke

Im Fall einer akuten, ausgeprägten nächtlichen Unruhe mit Selbstgefährdung wird eine medikamentöse Behandlung unerlässlich.
}

Dabei steht den Wirkungen der für diese Indikation gebräuchlichsten Pharmaka ihr unter Umständen erhebliches Nebenwirkungsprofil gegenüber. Dieses therapeutische Dilemma muss durch ein Abwägen der in > Tab. $\mathbf{3}$ zusammengefassten Vor- und Nachteile der jeweiligen Präparate gelöst werden. 
- Tab. 1 Ursachen, Differenzialdiagnosen, diagnostische Maßnahmen und therapeutische Interventionen für nächtliche Unruhezustände.

\begin{tabular}{|c|c|c|c|}
\hline Ursache & Differenzialdiagnosen z. B. & diagnostische Maßnahmen z. B. & therapeutische Intervention z. B. \\
\hline ängstliche Agitiertheit & $\begin{array}{l}\text { Angst vor fremder Umgebung, Dunkelheit } \\
\text { (Nyktophobie'), bevorstehender Operation, } \\
\text { Schlafneurose (Insomnophobie) }\end{array}$ & Exploration & $\begin{array}{l}\text { ggf. Rooming-in, Fenster, verhal- } \\
\text { tenstherapeutische Intervention } \\
\text { (z. B. Entspannungsverfahren) }\end{array}$ \\
\hline Schmerzen & $\begin{array}{l}\text { - internistisch: periphere arterielle Ver- } \\
\text { schlusskrankheit, Obstipation, Reflux, } \\
\text { Divertikulitis } \\
\text { - chirurgisch: Wundschmerz, Dekubitus } \\
\text { (z. B. lagerungsbedingt) } \\
\text { - orthopädisch: Arthrose, Lumbalgie } \\
\text { - urologisch: Blasenentleerungsstörung } \\
\text { - neurologisch: Spinalkanalstenose, periphere } \\
\text { Polyneuropathie, Burning Feet }\end{array}$ & $\begin{array}{l}\text { körperliche Untersuchung, } \\
\text { Labor }\end{array}$ & $\begin{array}{l}\text { Behandlung der körperlichen } \\
\text { Grunderkrankung, ggf. Physiotherapie, } \\
\text { ggf. Analgetika, ggf. gezielte } \\
\text { Pharmakotherapie }\end{array}$ \\
\hline $\begin{array}{l}\text { nächtlicher } \\
\text { Bewegungsdrang }\end{array}$ & $\begin{array}{l}\text { Restless-Legs-Syndrom (RLS), periodische } \\
\text { nächtliche Beinbewegungen (PLMS) }\end{array}$ & Anamnese, Polysomnografie & ggf. dopaminerge Therapie \\
\hline $\begin{array}{l}\text { Agrypnia excitata } \\
\text { (beständige motorische } \\
\text { Unruhe) }\end{array}$ & $\begin{array}{l}\text { fatale familiäre Insomnie (erbliche Prionener- } \\
\text { krankung), Morvan-Syndrom (Autoimmunen- } \\
\text { zephalitis) }\end{array}$ & $\begin{array}{l}\text { (je nach Grunderkrankung) } \\
\text { Anamnese, Elektroenzephalo- } \\
\text { grafie (EEG), Polysomnografie, } \\
\text { zerebrale Bildgebung, } \\
\text { Liquoranalytik }\end{array}$ & $\begin{array}{l}\text { (je nach Grunderkrankung) } \\
\text { Immunsuppression, Plasmapherese, } \\
\text { symptomatisch }\end{array}$ \\
\hline $\begin{array}{l}\text { Parasomnie, } \\
\text { pathologisches Erwachen }\end{array}$ & $\begin{array}{l}\text { Alptraum, Narkolepsie, Schlafwandeln, Pavor } \\
\text { nocturnus, verwirrtes Erwachen, REM-Schlaf- } \\
\text { störung (häufig bei Synukleinopathien, z. B. } \\
\text { bei DLB²) }\end{array}$ & Anamnese, Polysomnografie & $\begin{array}{l}\text { Aufklärung, nicht pharmakologische } \\
\text { Maßnahmen (z. B. Psychotherapie, } \\
\text { Meiden von Auslösern/Stressoren) } \\
\text { und (seltener) Pharmakotherapie }\end{array}$ \\
\hline internistisch & $\begin{array}{l}\text { Hypoglykämie, Hyperthyreose, Dehydratation, } \\
\text { Hyponatriämie, Urämie, Herzinsuffizienz, } \\
\text { nächtliches RR-Dipping, Fieber, Infektion, } \\
\text { „critical illness“, „frailty“ }\end{array}$ & $\begin{array}{l}\text { körperliche Untersuchung, } \\
\text { Labor }\end{array}$ & $\begin{array}{l}\text { Behandlung der internistischen } \\
\text { Grunderkrankung }\end{array}$ \\
\hline $\begin{array}{l}\text { Medikamente/Intoxikation/ } \\
\text { Entzug }\end{array}$ & $\begin{array}{l}\text { - Polypharmazie }{ }^{3} \text { (z. B. Kortison, Anticholi- } \\
\text { nergika, Benzodiazepine, Z-Substanzen, } \\
\text { Antipsychotika }{ }^{4} \text { ) } \\
\text { - Alkohol, Kaffee, Tee } \\
\text { - Delirium tremens }\end{array}$ & $\begin{array}{l}\text { Medikamenten- und } \\
\text { Suchtmittelanamnese }\end{array}$ & $\begin{array}{l}\text { Medikationsumstellung, } \\
\text { Entzugsbehandlung }\end{array}$ \\
\hline psychische Erkrankung & $\begin{array}{l}\text { agitierte Depression, Manie, Intelligenzmin- } \\
\text { derung, Autismus, Angsterkrankung, } \\
\text { Schizophrenie }\end{array}$ & $\begin{array}{l}\text { Anamnese, psychiatrische } \\
\text { Exploration }\end{array}$ & $\begin{array}{l}\text { Behandlung der psychischen } \\
\text { Grunderkrankung }\end{array}$ \\
\hline Verwirrtheitszustand, Delir & $\begin{array}{l}\text { - Schlafmittel- oder Alkoholentzug, seroto- } \\
\text { nerges Syndrom, anticholinerges Syndrom³ } \\
\text { - Demenz (z. B. gemischte Demenz, DLB², } \\
\text { Alzheimer-Demenz, Morbus Parkinson, } \\
\text { vaskuläre Enzephalopathie) } \\
\text { - Commotio/Contusio cerebri, zerebrale } \\
\text { Raumforderung }\end{array}$ & $\begin{array}{l}\text { Anamnese, psychiatrische } \\
\text { Exploration inkl. kurzer Testung, } \\
\text { ggf. apparative Diagnostik, z. B. } \\
\text { zerebrale Bildgebung }\end{array}$ & $\begin{array}{l}\text { delirogene Ursachen minimieren } \\
\text { (Grunderkrankung, Pharmaka), } \\
\text { reorientierende Maßnahmen } \\
\text { (u. a. Optimierung der Sinneswahr- } \\
\text { nehmung durch Brille, Hörgerät), } \\
\text { Rooming-in, antidelirante } \\
\text { Pharmakotherapie }\end{array}$ \\
\hline $\begin{array}{l}\text { aufgehobene zirkadiane } \\
\text { Rhythmik }\end{array}$ & Schlaf-Wach-Rhythmus-Störung & Anamnese, Aktometrie & Chronotherapie, Pharmakotherapie \\
\hline Epilepsie & nächtliche oder Aufwachanfälle & EEG, Polysomnografie & Antikonvulsiva \\
\hline \multicolumn{4}{|c|}{$\begin{array}{l}1 \text { Z. B. verstärkte Nachtangst bei Schlafmittelabhängigkeit oder Angst vor der Umgebung. } \\
2 \text { Demenz mit Lewy-Körperchen: Demenz plus Fluktuationen des Verlaufs oder visuelle Halluzination oder Rigor und Hypokinese oder Neuroleptika- } \\
\text { Überempfindlichkeit. } \\
{ }^{3} \text { Cave: Polypharmazie: jedes Medikament hat auch anticholinerge Eigenschaften. } \\
{ }^{4} \text { Cave: katatones Dilemma: jedes Antipsychotikum kann ein malignes neuroleptisches Syndrom (hypodopaminerg) auslösen, das schwer von einer (febrilen) } \\
\text { Katatonie (hyperdopaminerg) abgegrenzt werden kann. }\end{array}$} \\
\hline
\end{tabular}


- Tab. 2 Orientierende Untersuchung bei Verdacht auf kognitive Defizite und einen Verwirrtheitszustand (modifiziert nach [1]).

\begin{tabular}{|l|l|l|}
\hline \multirow{2}{*}{ Kriterien } & Test & Punkte \\
\hline \multirow{2}{*}{ Wachheit } & bei Ansprache oder Berührung keine angemessene Reaktion innerhalb 10 s & 4 \\
\hline Orientierung & Alter, Geburtsdatum, Ort, Jahr: 1 Fehler & 1 \\
\hline & $>1$ Fehler & 2 \\
\hline Aufmerksamkeit & Monate eines Jahres rückwärts: weniger als 7 & 1 \\
\hline & nicht durchführbar & 2 \\
\hline Fluktuation & Aufmerksamkeit, Wahrnehmung; Halluzinationen: ja & 4 \\
\hline $\begin{array}{l}\text { Beurteilung Summenwert: } \\
\text { 0: kognitive Defizite oder Delir unwahrscheinlich } \\
\text { 1-3: mögliche kognitive Defizite } \\
\text { > 3: Verwirrtheitszustand mit oder ohne dauerhafte kognitive Defizite möglich. }\end{array}$ \\
\hline
\end{tabular}

- Tab. 3 Pharmakotherapie der nächtlichen Unruhe bei Patienten mit demenziellen Erkrankungen.

\begin{tabular}{|c|c|c|c|}
\hline $\begin{array}{l}\text { Präparat/ } \\
\text { Dosierung }\end{array}$ & Indikation & Vorteil & Nachteil \\
\hline $\begin{array}{l}\text { Benzodiazepine/ } \\
\text { Benzodiazepin- } \\
\text { artige Substanzen }\end{array}$ & $\begin{array}{l}\text { " akute, sonst nicht beherrschbare } \\
\text { nächtliche Unruhe (v. a. bei Mul- } \\
\text { timorbidität, Angstzuständen) } \\
\text { - nächtliche Entzugssymptome } \\
\text { bei bereits vorhandener } \\
\text { Schlafmittel- oder Alkoholab- } \\
\text { hängigkeit }\end{array}$ & $\begin{array}{l}\text { - keine anticholinergen Nebenwir- } \\
\text { kungen } \\
\text { - (im Gegensatz zu Antipsychotika) } \\
\text { keine QTc-Zeit-Verlängerung [5] }\end{array}$ & $\begin{array}{l}\text { - unphysiologischer Schlaf } \\
\text { (reduzierter Tiefschlaf) } \\
\text { - verschlechtert Symptome einer } \\
\text { Demenz (z. B. Kognition, } \\
\text { Verwirrtheit) } \\
\text { - paradoxe Reaktion } \\
\text { - Abhängigkeit } \\
\text { - Tagesmüdigkeit } \\
\text { - Sturzgefahr }\end{array}$ \\
\hline $\begin{array}{l}\text { Quetiapin } \\
12,5-25 \mathrm{mg}\end{array}$ & Insomnie, nächtliche Unruhe & $\begin{array}{l}\text { - günstiges Rezeptorprofil (dosis- } \\
\text { abhängig kaum anticholinerg } \\
\text { und kaum dopaminerg) } \\
\text { - dosisabhängig wenig Überhang } \\
\text { am Folgetag }\end{array}$ & $\begin{array}{l}\text { - Cave: orthostatische Dysregulation } \\
\text { und QTc-Zeit-Verlängerung } \\
\text { - Auslösen/Verstärken eines RLS } \\
\text { möglich }\end{array}$ \\
\hline $\begin{array}{l}\text { Risperidon } \\
0,5-1,5 \mathrm{mg}\end{array}$ & $\begin{array}{l}\text { Behandlung von demenzassoziierten } \\
\text { Verhaltensauffälligkeiten einschließ- } \\
\text { lich nächtlicher Unruhe }\end{array}$ & $\begin{array}{l}\text { nachgewiesene Wirksamkeit bei der } \\
\text { Behandlung von Störungen des SWR } \\
\text { bei demenziellen Erkrankungen }\end{array}$ & $\begin{array}{l}\text { Cave bei DLB: Sturzgefahr wegen } \\
\text { extrapyramidal-motorischer } \\
\text { Nebenwirkungen }\end{array}$ \\
\hline $\begin{array}{l}\text { Mirtazapin } \\
7,5-15 \mathrm{mg}\end{array}$ & $\begin{array}{l}\text { Insomnie (bei komorbider } \\
\text { Depression) }\end{array}$ & $\begin{array}{l}\text { - kann bei (nicht dementen) älte- } \\
\text { ren Patienten die Nachtschlaf- } \\
\text { kontinuität verbessern [6] } \\
\text { - wirksam bei komorbider } \\
\text { Depression }\end{array}$ & $\begin{array}{l}\text { - Tagesmüdigkeit } \\
\text { - Appetit-/Gewichtszunahme } \\
\text { (erwünscht?) }\end{array}$ \\
\hline $\begin{array}{l}\text { Trazodon } \\
100-150 \mathrm{mg}\end{array}$ & $\begin{array}{l}\text { - Insomnie und/oder Sundowning } \\
\text { bei Demenz [3] } \\
\text { - (agitierte) Depression } \\
\text { (mit Insomnie) }\end{array}$ & $\begin{array}{l}\text { nachgewiesene Wirksamkeit für } \\
\text { Insomnie bei Demenz [7] }\end{array}$ & $\begin{array}{l}\text { - Cave: orthostatische Dysregulation, } \\
\text { Herzrhythmusstörungen } \\
\text { - Blutbildveränderungen }\end{array}$ \\
\hline $\begin{array}{l}\text { Melatonin } \\
2-4 \mathrm{mg}\end{array}$ & $\begin{array}{l}\text { Insomnie bei > } 55 \text { Jahre, Blindheit, } \\
\text { Jetlag }\end{array}$ & $\begin{array}{l}\text { Hinweise für Wirksamkeit bei } \\
\text { Störung des SWR bei Demenz [8] }\end{array}$ & $\begin{array}{l}\text { - lange Wirklatenz } \\
\text { - schwach hypnotisch }\end{array}$ \\
\hline $\begin{array}{l}\text { Clomethiazol } \\
\text { (Distraneurin) }\end{array}$ & (Alkohol-) Entzugsdelir & stark sedierend und antidelirant & $\begin{array}{l}\text { - Atemdepression } \\
\text { - Zunahme Bronchialsekretion } \\
\text { - Abhängigkeit } \\
\text { - Überwachung notwendig }\end{array}$ \\
\hline
\end{tabular}


Beruhigende, aufmerksame Zuwendung sichern, sichern, sichern - auch sich selbst!

Re-Orientierung durch Hörgerät, Brille, Uhr, Lichtregie, Fenster, Mobilisierung, ...

$\downarrow$

Symptomatisch

a) ggf. Reduktion anticholinerger, delirogener Substanzen

b) Auswahl geeigneter Psychopharmaka (Tabelle 3)

- Abb. 1 Nichtpharmakologische Interventionen sind notwendige Voraussetzungen zur Einleitung einer gezielten Therapie und weitergehenden Diagnostik, die parallel auf Symptome und Ursachen zielen.

Im akuten Notfall sind - gerade bei bereits süchtigen Patienten - Benzodiazepine in der niedrigsten notwendigen Dosis für die kürzeste notwendige Zeit geeignet. Insbesondere bei älteren Patienten verbietet sich darüber hinaus die Anwendung dieser „Schlafmittel“ aufgrund ihrer schlafkorrumpierenden Wirkung, der Sturzgefahr und des hohen Abhängigkeitspotenzials [9].

Antipsychotika mit starker Dopamin-Rezeptorblockade (Haloperidol, Risperidon, Olanzapin, Aripiprazol) sind aufgrund ihrer extrapyramidal-motorischen Nebenwirkungen für Patienten mit Synukleinopathien (Dopamin-Mangelerkrankungen) kontraindiziert [4]. Stattdessen stehen Antipsychotika mit geringerer antidopaminerger Wirkung wie Quetiapin zur Verfügung.

Bei Demenz mit Lewy-Körperchen kann zur Akutbehandlung nächtlicher Unruhezustände zunächst niedrig dosiert Quetiapin (z. B. 12,5-25 mg, Cave: Hypotonie) verordnet werden. Mittelfristig kann dann ein Ausgleich des cholinergen Defizits durch Gabe eines Acetylcholinesterase-Hemmers (z. B. Rivastigmin-Pflaster 4,6 mg/24 h) angestrebt werden.

\section{KERNAUSSAGEN}

- Für nächtliche Unruhezustände bei älteren Patienten gilt: Sie treten häufig, aber nicht ausschließlich im Rahmen von demenziellen Erkrankungen auf.

- Viele weitere Störungen müssen als Ursache in Betracht gezogen werden: internistische (z. B. Schmerzen, Stoffwechselerkrankung), neurologi- sche (z. B. Basalganglien-Störung), psychiatrische (z. B. Sucht- oder Entzugssymptomatik) und schlafmedizinische (z. B. Nyktophobie oder REM-Parasomnie).

- Eine eindeutige differenzialdiagnostische Abgrenzung zwischen Delir und Demenz ist nicht immer möglich und sinnvoll, da vorbestehende kognitive Störungen den wichtigsten Risikofaktor für einen Verwirrtheitszustand darstellen.

- Die Akuttherapie beinhaltet: beruhigende, aufmerksame Zuwendung, Absetzen einer anticholinergen Medikation, symptomatische pharmakologische Intervention sowie kausale Therapie zugrunde liegender medizinischer Ursachen.

- Benzodiazepine dürfen aufgrund ihres Intoxikations- und Abhängigkeitspotenzials und möglicher paradoxer Reaktionen nur in Ausnahmefällen (z. B. Abhängigkeit, ausgeprägte Selbstgefährdung), nur niedrig dosiert und kurzzeitig verordnet werden.

- Stattdessen wird, besonders bei komorbiden, depressiven oder psychotischen Symptomen, eine Pharmakotherapie mit Quetiapin oder Mirtazapin empfohlen.

\section{Interessenkonflikt}

Die Autorinnen/Autoren geben an, dass kein Interessenkonflikt besteht.

\section{Autorinnen/Autoren}

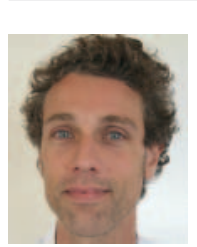

\section{Dirk Schwerthöffer}

Dr. med., Facharzt für Psychiatrie und Psychotherapie, Somnologe DGSM. Oberärztliche Tätigkeit in der Klinik und Poliklinik für Psychiatrie und Psychotherapie der Technischen Universität München in den Bereichen Intensivpsychiatrie und Schlafmedizin.

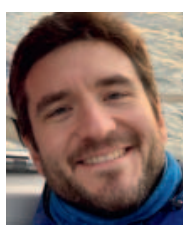

\section{Bastian Fatke}

Dr. med., Oberarzt und Leiter der Poliklinik und Institutsambulanz und des Konsildienstes sowie Lehrverantwortlicher, Klinik und Poliklinik für Psychiatrie und Psychotherapie der Technischen Universität München, Klinikum rechts der Isar.

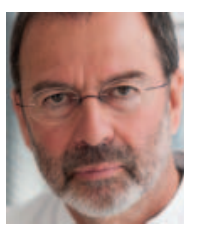

\section{Hans Förstl}

Univ.-Prof. Dr. med., Facharzt für Neurologie und Psychiatrie. Habilitation in Psychiatrie und Psychotherapie an der Universität Heidelberg. Seit 1997 Direktor der Klinik und Poliklinik für Psychiatrie und Psychotherapie der Technischen Universität München, Klinikum rechts der Isar. 
Korrespondenzadresse

Dr. med. Dirk Schwerthöffer

Klinik und Poliklinik für Psychiatrie und Psychotherapie der TU-München

Ismaninger Straße 22

81675 München

Dirk.Schwerthoeffer@mri.tum.de

\section{Literatur}

[1] Bellelli G, Morandi A, Davis DH et al. Validation of the 4AT, a new instrument for rapid delirium screening: a study in 234 hospitalised older people. Age Ageing 2014; 43: 496-502. doi:10.1093/ageing/afu021

[2] Schwerthöffer D, Förstl H. Störungen des Schlaf-Wach-Rhythmus bei demenziellen Erkrankungen. DNP 2020; 21: 18-22. doi:10.1007/s15202-019-0002-0

[3] Ringman JM, Schneider L. Treatment options for agitation in dementia. Curr Treat Options Neurol 2019; 21: 30. doi:10.1007/s11940-019-0572-3

[4] Deutsche Gesellschaft für Psychiatrie und Psychotherapie, Psychosomatik und Nervenheilkunde (DGPPN) und Deutsche Gesellschaft für Neurologie (DGN). S3-Leitinie „Demenzen“. Januar 2016. Im Internet (Stand: 19.03.2020): http://www.awmf.org/leitlinien/detail/II/038-013.html
[5] Schwerthöffer D, Forstl ], Fatke B. Antipsychotische Pharmakotherapie bei deliranten Syndromen - Nur zeitlich begrenzt, symptomorientiert und unter Beachtung der QTc-Zeit. MMW Fortschr Med 2019; 161: 50-52. doi:10.1007/s15006-019-1009-3

[6] Rothschild-Fuentes B, Roche A, Jimenez-Genchi A et al. Effects of mirtazapine on the sleep wake rhythm of geriatric patients with major depression: an exploratory study with actigraphy. Pharmacopsychiatry 2013; 46: 59-62. doi:10.1055/s-00321323655

[7] Grippe TC, Goncalves BS, Louzada LL et al. Circadian rhythm in Alzheimer disease after trazodone use. Chronobiol Int 2015; 32: 1311-1314. doi:10.3109/07420528.2015.1077855

[8] Cardinali DP, Furio AM, Brusco LI. The use of chronobiotics in the resynchronization of the sleep/wake cycle. Therapeutical application in the early phases of Alzheimer's disease. Recent Pat Endocr Metab Immune Drug Discov 2011; 5: 80-90. doi:10.2174/187221411799015354

[9] Beland SG, Preville M, Dubois MF et al. The association between length of benzodiazepine use and sleep quality in older population. Int J Geriatr Psychiatry 2011; 26: 908-915. doi:10.1002/ gps.2623

Bibliografie

DOI https://doi.org/10.1055/a-1124-2374

Dtsch Med Wochenschr 2020; 145: 634-638

(c) Georg Thieme Verlag KG, Stuttgart · New York ISSN 0012-0472 